\title{
Receptor Discordance of Metastatic Breast Cancer Depending on the Molecular Subtype
}

\author{
Iris Holzer Alex Farr Yen Tan Christine Deutschmann Carmen Leser \\ Christian F. Singer \\ Breast Health Center, Division of Gynecology and Gynecologic Oncology, Department of Obstetrics and \\ Gynecology, Medical University of Vienna, Vienna, Austria
}

\section{Keywords}

Breast cancer $\cdot$ Metastases $\cdot$ Receptor status $\cdot$ Personalized medicine

\begin{abstract}
Introduction: Almost 30\% of all women with early-stage breast cancer develop metastases. Treatment of metastatic disease is often based on the immunohistochemical information of the primary tumor, despite possible discordance of the hormone and Her2 receptor status. Objectives: The aim of this study was to compare the receptor status of the primary tumor with the metastasis, and to evaluate for receptor discordance with regard to the molecular subtype, receptor status, and the localization of the metastases. Methods: We retrospectively analyzed the data of all consecutive women with metastatic breast cancer, who underwent treatment at the Medical University Vienna between 2009 and 2016. Associations were calculated using the $x^{2}$ or Fisher's exact test; years from primary diagnosis to metastatic disease were calculated using the Kaplan-Meier method. $R \boldsymbol{e}$ sults: We identified 213 metastatic breast cancer patients, of whom 67 (31.5\%) showed a discordant receptor status. Out of 32 patients with luminal A subtype, 14 (43.8\%) had a switch of at least one receptor; 27 of 53 patients (50.9\%) with luminal B subtype and 21 of 32 patients (65.6\%) with Her2+
\end{abstract}

subtype showed receptor discordance; for triple-negative disease, 5 of 19 patients (36.3\%) had a switch of at least one receptor. In 63 samples of bone metastases, 13 (20.6\%) had discordant estrogen receptor status $(p=0.04)$. In 55 samples of bone metastases, 35 (63.3\%) had discordant Her2 status $(p=0.002)$. Conclusions: Our data show high rates of receptor discordance in metastatic breast cancer. Apart from the primary tumor, the immunohistochemical receptor status of the metastasis needs to be verified. This can lead to a change in treatment and prognosis.

๑) 2020 S. Karger AG, Basel

\section{Introduction}

Nearly one-third of patients with early-stage breast cancer at the time of first diagnosis suffer from metastatic disease during lifetime [1]. About 7\% of patients with initially diagnosed breast cancer already present with metastatic disease at time of first diagnosis [2]. Despite the interval of many years between primary diagnosis and occurrence of metastases in many cases, the therapeutic management of metastatic disease is often based on the receptor status of the primary lesion. However, differences between the receptor status of the primary tumor and the metastases exist [3]. 
Knowledge of a discordant receptor status may lead to a change in therapeutic decisions [4]. Due to different treatment strategies and prognosis, the importance of a discordant hormone receptor (HR) status of primary tumor and metastases has already been recognized. According to the available literature, discordance rates of 10 $40 \%$ have been reported [5]. Discordance of the estrogen receptor (ER) and progesterone receptor (PR) status was reported to occur in $29 \%$, respectively, whereas discordance of the Her2 status was reported to occur in $4 \%$ of all cases. Most frequently, the receptor status switched from positive to negative, which of course requires adjustment of treatment strategies [6].

Molecular breast cancer subtypes are associated with different models of metastatic behavior and provide different information about the prognosis [7-9]. It has been demonstrated that $3.28,1.52,1.2$, and $0.35 \%$ of patients with firstly diagnosed breast cancer had bone, lung, liver, and brain metastasis, respectively. The $\mathrm{HR}+/ \mathrm{Her} 2+$ subtype correlates with an increased risk of bone metastasis, whereas HR-/Her2+ does not; Her2+ subtypes, irrespective of the HR status, show significantly higher rates of liver, brain, and lung metastases. Triple-negative (TN) tumors are known to have a higher risk of brain, liver, and lung metastasis, as well as decreased risk of bone metastasis [10]. The longest rate of distant metastasis-free survival was demonstrated in breast cancer patients with luminal A subtype, and the shortest was reported for the Her2+ subtype. In addition, luminal A tumors are associated with bone only metastases, and tumors of the TN subtype have higher rates of brain metastases [7].

The aim of the present study was to establish a large dataset of metastatic breast cancer patients and to evaluate, whether there is a discordance of the receptor status between the primary tumor and the metastasis, depending on the molecular subtype and the localization of the metastatic lesion. To our knowledge, this study is the first to evaluate this topic.

\section{Materials and Methods}

The study was conducted on the Medical University Vienna, Department of Obstetrics and Gynecology, Division of Gynecology and Gynecological Oncology, Breast Health Center. In a retrospective manner, data of all patients who underwent treatment for metastatic breast cancer between 2009 and 2016 at our Department were obtained. The clinical and histopathological information was drawn from the patient-related hospital information system, the AKIM software (SAP Inc., Walldorf, Baden-Württemberg, Germany). All data were encoded using Excel (Microsoft Corp., Redmond, WA, USA) and SPSS Statistics version 23.0 (IBM, New York, NY, USA).

Descriptive statistics were calculated, and estimates were reported in frequencies and percentages. The association between the molecular subtype and the age at diagnosis, tumor grade, histology type, survival status, and the localization of the metastatic
Table 1. Molecular subtype of the primary tumor in 213 patients

\begin{tabular}{lrr}
\hline Molecular subtype & $N$ & $\%$ \\
\hline Luminal A & 45 & 21.1 \\
Luminal B & 66 & 31 \\
Her2+ & 40 & 18.7 \\
Triple negative & 31 & 14.6 \\
N/A & 31 & 14.6 \\
\hline Total & 213 & 100 \\
\hline
\end{tabular}

N/A, not available.

Table 2. Receptor discordance of the molecular subtype in 136 patients

\begin{tabular}{lrcc}
\hline \multirow{2}{*}{ Subtype } & \multicolumn{3}{l}{ Receptor discordance } \\
\cline { 2 - 4 } & yes & no & total \\
\hline Luminal A, $n(\%)$ & $14(43.8)$ & $18(56.3)$ & 32 \\
Luminal B, $n(\%)$ & $27(50.9)$ & $26(49.1)$ & 53 \\
Her2+, $n(\%)$ & $21(65.6)$ & $11(34.4)$ & 32 \\
TN, $n(\%)$ & $5(26.3)$ & $14(73.7)$ & 19 \\
\hline All, $n(\%)$ & $67(49.3)$ & $69(50.7)$ & $136(100)$ \\
\hline
\end{tabular}

lesion were evaluated using the $\chi^{2}$ or Fisher's exact test (if sample size $<5)$. Years from primary to metastatic diagnosis were calculated using the Kaplan-Meier method. The association between HR discordance and metastasis was also evaluated using the $\chi^{2}$ or Fisher's exact test. HR discordance was defined as a switch from either direction (i.e., negative to positive, or positive to negative).

The molecular subtype was classified as follows: a positive receptor status of ER and/or PR and a negative Her2 status with low proliferation was considered as luminal A subtype; a positive receptor status of ER and/or PR and a negative Her2 status with high proliferation were considered as luminal B subtype; non-luminal with a positive Her2 receptor status and a negative ER and PR status, as well as luminal with a positive Her2 status and ER and/or PR positive, were considered as Her2 subtype; and a negative Her2, ER, and PR status was considered as the basal-like or TN subtype. The Her2 subtype was not further categorized as luminal or nonluminal.

\section{Results}

Between 2009 and 2016, a total of 213 patients with metastatic breast cancer were cared for at our Department and fulfilled the inclusion criteria for further analysis. The mean ( \pm standard deviation) age at diagnosis of the primary tumor was $53 \pm 12.3$ years. The molecular subtypes of the primary tumor were luminal A in 45 cases $(21.1 \%)$, luminal B in 66 cases ( $31 \%)$, Her $2+$ in 40 cases (18.7\%), and TN in 31 cases (14.6\%). In another 31 cases $(14.6 \%)$, there was no information about the molecular subtype available (Table 1). 
Table 3. Receptor status of 16 patients with multiple metastatic lesions

\begin{tabular}{|c|c|c|c|c|c|c|c|c|c|c|c|c|c|}
\hline \multirow[t]{2}{*}{ Patient } & \multicolumn{4}{|c|}{ Primary breast cancer } & \multicolumn{4}{|c|}{ Metastatic lesion, biopsy 1} & \multicolumn{4}{|c|}{ Metastatic lesion, biopsy 2} & \multirow{2}{*}{$\begin{array}{l}\text { Discor- } \\
\text { dance }^{a}\end{array}$} \\
\hline & ER & PR & Her2 & Ki67 (\%) & localization & ER & PR & Her2 & localization & ER & PR & Her2 & \\
\hline 1 & pos. & neg. & neg. & 30 & Bone & pos. & neg. & neg. & Skin & pos. & neg. & neg. & no \\
\hline 2 & pos. & pos. & neg. & 5 & Bone & pos. & neg. & neg. & Liver & pos. & neg. & neg. & yes \\
\hline 3 & pos. & neg. & neg. & 10 & Bone & pos. & neg. & neg. & Liver & pos. & neg. & neg. & no \\
\hline 4 & pos. & pos. & neg. & 30 & Bone & pos. & pos. & neg. & Cervix & pos. & pos. & neg. & no \\
\hline 5 & pos. & pos. & neg. & 40 & Bone & neg. & neg. & pos. & Liver & pos. & neg. & neg. & yes \\
\hline 6 & pos. & pos. & neg. & 5 & Skin & pos. & pos. & neg. & Vulva & pos. & pos. & neg. & no \\
\hline 7 & neg. & neg. & pos. & N/A & Liver & pos. & pos. & pos. & Bone & pos. & neg. & pos. & yes \\
\hline 8 & pos. & neg. & neg. & 80 & Liver & pos. & neg. & neg. & Brain & pos. & neg. & neg. & no \\
\hline 9 & pos. & neg. & neg. & 5 & Liver & pos. & pos. & neg. & Bone & neg. & neg. & neg. & yes \\
\hline 10 & neg. & neg. & pos. & 30 & Liver & neg. & neg. & pos. & Skin & neg. & neg. & pos. & no \\
\hline 11 & pos. & pos. & pos. & 10 & Peritoneum & pos. & neg. & neg. & Peritoneum & pos. & neg. & neg. & yes \\
\hline 12 & pos. & neg. & pos. & 20 & Skin & pos. & neg. & neg. & Pleura & pos. & N/A & $\mathrm{N} / \mathrm{A}$ & yes \\
\hline 13 & pos. & pos. & neg. & 90 & Skin & pos. & neg. & neg. & Bone & pos. & pos. & neg. & yes \\
\hline 14 & pos. & pos. & neg. & 40 & Liver & pos. & neg. & neg. & Bone & pos. & neg. & neg. & yes \\
\hline 15 & neg. & neg. & neg. & 70 & Bone & neg. & neg. & neg. & Liver & neg. & neg. & neg. & no \\
\hline 16 & pos. & neg. & neg. & 90 & Cartilage & pos. & neg. & neg. & Skin & pos. & neg. & neg. & no \\
\hline
\end{tabular}

N/A, not available; ER, estrogen receptor; PR, progesterone receptor; pos., positive; neg., negative. ${ }^{\text {a }}$ Defined as discordance of receptor status between primary tumor and any metastatic lesion on immunohistochemical analysis.

The metastatic lesions were localized as follows: liver in 78 cases $(36.6 \%)$, lung in 53 cases $(24.9 \%)$, bone in 100 cases $(46.9 \%)$, brain in 21 cases $(9.9 \%)$, skin in 48 cases (22.5\%), and others in 62 cases (29.1\%; multiple localizations were allowed). Other metastatic lesions included the pleura, peritoneum, ovaries, adrenal glands, cervix, urinary bladder, vagina and vulva, mediastinum, colon, orbit, stomach, and parotid gland.

All records of the pathological sample of the primary tumor and of the biopsy of all groups of metastatic lesions were compared and analyzed. Overall, 135 samples with available receptor status of the primary tumor and the metastatic lesion were analyzed. Discordance of the receptor status was found among all molecular subtypes, as shown in Table 2.

A total of 136 immunohistochemical (IHC) records of the primary lesion and the biopsy of the metastatic lesion were available. Of this cohort, 67 samples (49.3\%) showed a discordance of any receptor status and 69 (50.7\%) showed no discordance of the receptor status.

Of 32 samples with a luminal A subtype of the primary tumor, 14 samples $(43.8 \%)$ had a discordance of any receptor status and 18 samples $(56.3 \%)$ had no discordance. In the cohort of 53 samples of the luminal B subtype, 27 samples (50.9\%) revealed discordance and in 26 samples (49.1\%), there was no discordance of any receptor status. The Her2+ molecular subtype was present in 32 samples. Out of these, 21 (65.6\%) IHC records had any discordance of the receptor status and 11 (34.4\%) had no discordance. Nineteen samples were considered to be TN and out of these, 5 (26.3\%) showed any discordance and
$14(73.7 \%)$ showed no discordance. Sixteen of 213 patients had available IHC data from multiple metastases, as shown in Table 3.

The discordance of any receptor status from negative to positive or from positive to negative was statistically significantly different in the groups of the molecular subtypes luminal A, luminal B, Her2+ and TN ( $p=0.05)$. A discordant ER status was observed in 19 patients (14.1\%), whereas 39 patients $(29.1 \%)$ had a discordant PR status, and 29 patients $(23.4 \%)$ had a discordant Her2 status. There was a statistically significant difference in the contribution of patients with bone metastases and the different molecular subtype $(p=0.02)$. Luminal A was the most frequently associated molecular subtype with bone metastatic disease. Molecular subtypes and localization of metastatic lesions are listed in Table 4.

In 63 samples with bone metastatic disease, 13 (20.6\%) showed discordance of the ER status $(p=0.04)$. In 55 samples with bone metastases and initial Her2+ status, 35 (63.6\%) showed discordance of the Her2 status $(p=$ 0.002). In 35 samples of skin metastatic disease, 33 (94.3\%) showed no discordance of the Her2 status; in 2 cases (5.7\%), we found a discordant Her2 status between the primary tumor and the skin metastasis.

With regard to local recurrence, we found a statistically significant association between the primary tumor and locally recurrent lesion, with regard to the $\operatorname{ER~(~} p=$ $0.002), \operatorname{PR}(p=0.024)$, and HER2 receptor status $(p<$ $0.001)$. Discordant rates were $19.3,32.2$, and $13.8 \%$ for ER, PR, and HER2, respectively. 
Table 4. Molecular subtype and localization of metastases in 182 patients

\begin{tabular}{|c|c|c|c|c|c|c|}
\hline & \multicolumn{6}{|c|}{ Molecular subtype } \\
\hline & luminal $\mathrm{A}$ & luminal B & Her $2+$ & $\mathrm{TN}$ & total & $p$ \\
\hline \multicolumn{7}{|l|}{ Liver } \\
\hline No, $n(\%)$ & $31(68.9)$ & $41(62.1)$ & $26(65)$ & $20(64.5)$ & $118(64.8)$ & \\
\hline Yes, $n(\%)$ & $14(31.1)$ & 25 (37.9) & $14(35)$ & $11(35.5)$ & $64(35.2)$ & 0.91 \\
\hline \multicolumn{7}{|l|}{ Lung } \\
\hline No, $n(\%)$ & $33(73.3)$ & $51(77.3)$ & $27(67.5)$ & $22(71)$ & $133(73.1)$ & \\
\hline Yes, $n(\%)$ & $12(26.7)$ & $15(22.7)$ & $13(32.5)$ & $9(29)$ & $49(26.9)$ & 0.73 \\
\hline \multicolumn{7}{|l|}{ Bone } \\
\hline No, $n(\%)$ & $16(35.6)$ & $38(57.6)$ & $22(55)$ & $22(71)$ & $98(53.8)$ & \\
\hline Yes, $n(\%)$ & $29(64.4)$ & $28(42.4)$ & $18(45)$ & $9(29)$ & $84(46.2)$ & 0.02 \\
\hline \multicolumn{7}{|l|}{ Brain } \\
\hline No, $n(\%)$ & $43(95.6)$ & $62(93.9)$ & $34(85)$ & $25(80.6)$ & $164(90.1)$ & \\
\hline Yes, $n(\%)$ & $2(4.4)$ & $4(6.1)$ & $6(15)$ & $6(19.4)$ & $18(9.9)$ & 0.08 \\
\hline \multicolumn{7}{|l|}{ Skin } \\
\hline No, $n(\%)$ & $38(84.4)$ & $50(75.8)$ & $30(75)$ & $22(71)$ & $140(76.9)$ & \\
\hline Yes, $n(\%)$ & $7(15.6)$ & $16(24.2)$ & $10(25)$ & 9 (29) & $42(23.1)$ & 0.53 \\
\hline \multicolumn{7}{|l|}{ Other } \\
\hline No, $n(\%)$ & $33(73.3)$ & $38(57.6)$ & $30(75)$ & $24(77.4)$ & $125(68.7)$ & \\
\hline Yes, $n(\%)$ & $12(26.7)$ & $28(42.4)$ & $10(25)$ & $7(22.6)$ & $57(31.3)$ & 0.11 \\
\hline
\end{tabular}

\section{Discussion}

Apart from the confirmation of breast cancer recurrence, biopsies of metastatic reasons are taken to confirm the receptor status of the metastatic lesion. Our study underlines the idea of evaluating the receptor status of metastases by biopsies at the time of the recurrence of the disease.

Biopsies are rarely performed to confirm the diagnosis of metastatic disease, even in the case of an interval of many years from initial diagnosis to relapse. Nevertheless, therapeutic decisions on the systemic therapy for advanced breast cancer with antihormonal therapy, cytotoxic or targeted agents are often based on the biological information of the patients' original receptor status of the biopsy or surgery at time of the initial diagnosis. However, certain characteristics of the receptor status such as ER, PR, and Her2 status may change. To avoid overtreatment, undertreatment, or inadequate treatment, biopsies of the suspected metastatic lesion should be performed in patients with suspected metastatic disease [11].

In a large European study, a potential discordance of the subtype of metastatic breast cancer was observed [12]. Out of 132 patients with primary breast cancer, 74 presented with distant metastases. The most frequent localization of distant metastatic lesions in this study was bone, followed by liver and lung. The discordance rate of single receptor switch was $20 \%$ for ER, $37 \%$ for PR and $3 \%$ for the Her 2 receptor. Additionally, the study group that changed subtype had a longer mean time to recurrence compared to the study group with no change of the subtype ( 44.9 vs. 36.9 months; $p=0.16$ ). The authors of this study did not find a statically significant association between the localization of the metastatic lesions, the type of surgery or neoadjuvant therapy, and the change of the molecular subtype. The total rate of a change of subtype was $23.5 \%$, with the most common switch from luminal A to TN. The results of the most frequent metastatic lesions stand in accordance to our data, as we observed bone (46.9\%) as the most frequent metastatic site, followed by liver $(36.6 \%)$ and lung (24.9\%). We observed a similar rate of any receptor status discordance (49.3\%), but in our collective, a statistically significant association of any receptor switch with metastasis localization was observed. This fact could be explained by the lower number of included metastatic patients in the study of McAnena et al. [12] that could lack statistical power.

A large study from Spain was one of the first prospective studies evaluating the discordance rate of the biomarker status for the different subtypes of breast cancer. Out of a cohort of 184 included patients with metastatic breast cancer, the discordance rate of the Her2 status between primary tumor and metastatic lesion was $16 \%$ (95\% CI 10.7-23.4), with a similar distribution in gain or loss of Her 2 overexpression. In this large study, the rate of receptor discordance was highest in the HR-positive subtype, while only 1 patient with TN breast cancer and 
2 patients with the Her2+ subtype showed a switch of their Her2 status. For ER, the discordance rate was 21\% (95\% CI 15.7-28.9). The discordance rate for PR was 35\% (95\% CI 27.5-42.8). Overall, the conversion rate of the HR was reported to be $15 \%$ with equal distribution from positive to negative and vice versa. In 36 tumor samples (6\%), the molecular subtype between the tumor at initial diagnosis and the metastasis showed discordance. The switch from HR positive to Her2+ tumors (9\%) was the most common one. Other usual switches were from HR positive to TN tumors (5\%), and vice versa (4\%), and changes between TN and Her2+ tumors in either direction [13]. Thus, the results of our study with a comparable sample size of included cases with IHC records of the primary tumor and metastatic lesions are not in accordance with the results of that study.

In our study, a discordance rate of the Her2 receptor status was observed in 21 of $32(65.6 \%)$ cases. But our data showed the TN subtype as the less frequent switch, demonstrating a switch of any receptor status in 5 of 19 cases $(26.3 \%)$. As we agree that a discordance of any receptor occurs less often in the TN subtype, it is difficult to define a reason for the more commonly observed discordance of the Her2 receptor status that we found. The high number of observed discordances of the Her2 receptor in our study population might be explained by the fact that in many cases, the Her2 analysis was performed by both technical methods: fluorescence in situ hybridization (FISH) and IHC staining. It may be that we had a very accurate evaluation of the Her2 status, due to the double evaluation by two different methods.

Potential mechanisms and etiologies for the reported discordance between primary tumor and metastasis are mostly likely manifold. First of all, technical causes have to be considered, as variability in the reproducibility and accuracy of IHC staining exists [14]. Furthermore, the various methods of retrieving tissue for IHC assessment exist, including fine needle aspiration, core biopsy, and surgical extraction of the lesion. Another cause that might contribute to the receptor status discrepancy is a selection and growth of malignant cells, because of a treatment that results in a more aggressive phenotype. Targeted killing of HR-positive cancer cells by adjuvant antihormonal therapy might lead to a selection of untreated ER- and PR-negative cells, which might again be the cause for metastatic relapse in terms of a biological drift. Moreover, the presence of HR-negative cancer cells might have an effect of the clonal selection among cancer cells, resulting in mosaicism within the ER expression. The switch of the receptor status might contribute to new gained advantages for the tumor cell with a possible increased capability for invasion as endocrine and growth factor signaling pathways implicate invasion and metastatic dissemination $[15,16]$.
Heterogeneity between primary cancer and metastatic lesion might be based on recently gained biological characteristics that enable tumor cells to wander through the circulatory and lymph system, in order to metastasize to distant organs $[15,16]$. Additionally, a more difficult IHC staining process in some metastatic tissues, especially in the bone, might play a role, pretending the loss of any receptor because of methods for decalcifying the bone biopsies [17]. Available data suggest that the common methods for decalcifying the bones do not hamper the analysis of tumor phenotype and do not affect the IHC evaluation [18-20]. Especially the Her2 receptor status in bone metastases has to be considered in this context. The Her2 receptor status of bone metastases can either be analyzed by FISH (for gene amplification) or by IHC (for protein overexpression). Both methods are considered to have high levels of accuracy and provide equal results [17].

The conventional model of breast cancer progression is based on the paradigm that breast cancer passes through several stages from an in situ stage to an invasive stage, followed by dissemination to the lymph nodes and distant organs. In contrast to this model, Narod and Sopik [21] proposed an alternative model of breast cancer development and metastasis, describing the initial metastatic potential of a small pool of cancer stem cells, which disseminate synchronously through several routes to the breast stroma, to the lymph nodes and to distant organs. The alternative model is based on empirical facts about large observational studies on patients with ductal carcinoma in situ (DCIS), showing that radical mastectomy did not reduce the risk of dying from breast cancer but the risk of local recurrence. The authors interpret local recurrence after invasive cancer as a marker of cancer aggressiveness, but not as a conduit of cancer spread, concluding that local recurrence does hence not metastasize, and that DCIS has inherent malignant potential [21]. Our study primarily focused on the conventional model, postulating new ideas on the common understanding of breast cancer metastasis.

We are aware that our study has several limitations. Apart from the retrospective design, IHC evaluation was performed for clinical use and not primary for the purpose of our study. There might be a discrepancy between pathologists who evaluated the IHC. Some metastatic sites are rarely observed in breast cancer patients, and the low number of included cases might lack statistical power. In rare cases, a biopsy of the metastatic lesions could not have been performed due to the low performance status or comorbidities of the individual patient, or due to missing technical abilities; for example, small metastases of the liver or lung, that are not accessible for CT scan or MRI-guided biopsies. Theoretically, these rare cases with metastases without IHC evaluation could have biased our 
results. Apart from the available literature reporting that IHC from bone metastases are not more difficult to evaluate than those from other tissues, and that the decalcification process does not alter the results, we are unable to exclude a difference of the IHC in different tissues. Lastly, we were unable to evaluate whether there was a loss or a gain of the receptor status, evaluating just the receptor discordance, and we did not incorporate genetic tests of the cancer tissues in our study protocol.

The strengths of our study include a relatively large dataset of patients with metastatic breast cancer; in a considerable number of patients, a biopsy of the metastatic lesion was performed, and histopathological records of the primary breast tumor and the metastatic lesion were available for statistical analysis. Since patients that were cared for at our Center were followed at frequent intervals, diagnosis and biopsy of the metastatic lesion were generally performed at an early time point.

\section{Conclusion}

The present study emphasizes the importance of the molecular subtype of the initial breast cancer and the metastatic lesion for optimizing personalized therapy in each individual cancer patient [22-24]. Apart from the IHC assessment of the primary tumor, IHC of the metastatic lesion needs to be evaluated, considering that $31.5 \%$ of all patients show discordance between the primary and metastatic lesions. Although this information might involve additional biopsies, it is crucial to avoid unnecessary treatment and to optimize prognosis.

\section{Statement of Ethics}

All procedures performed in studies involving human participants were in accordance with the ethical standards of the institutional research committee of the Medical University of Vienna (registration number 1841/2014) and with the 1964 Helsinki Declaration and its later amendments or comparable ethical standards. Due to the retrospective study design and following the waiver of the ethical committee, informed consent was not obtained.

\section{Disclosure Statement}

The authors have no conflicts of interest to declare.

\section{Funding Sources}

None.

\section{Author Contributions}

I.H., Y.T., and C.F.S. designed the study; C.D., C.L., I.H., and A.F. collected the data; Y.T. and AF performed the statistical analyses; I.H., A.F., Y.T., and C.F.S. wrote the paper. All authors approved the final version of the paper.

\section{References}

1 O'Shaughnessy J. Extending survival with chemotherapy in metastatic breast cancer. Oncologist. 2005;10(S3 Suppl 3):20-9.

2 Redig AJ, McAllister SS. Breast cancer as a systemic disease: a view of metastasis. J Intern Med. 2013 Aug;274(2):113-26.

3 Ibrahim T, Farolfi A, Scarpi E, Mercatali L, Medri L, Ricci M, et al. Hormonal receptor, human epidermal growth factor receptor-2, and Ki67 discordance between primary breast cancer and paired metastases: clinical impact. Oncology. 2013;84(3):150-7.

4 Heitz F, Barinoff J, du Bois O, Hils R, FisselerEckhoff A, Harter P, et al. Differences in the receptor status between primary and recurrent breast cancer-the frequency of and the reasons for discordance. Oncology. 2013; 84(6):319-25.

5 Pusztai L, Viale G, Kelly CM, Hudis CA. Estrogen and HER-2 receptor discordance between primary breast cancer and metastasis. Oncologist. 2010;15(11):1164-8.

6 Curtit E, Nerich V, Mansi L, Chaigneau L, Cals L, Villanueva C, et al. Discordances in estrogen receptor status, progesterone receptor status, and HER2 status between primary breast cancer and metastasis. Oncologist. 2013 Jun;18(6):667-74.
7 Molnár IA, Molnár BA, Vízkeleti L, Fekete K, Tamás J, Deák P, et al. Breast carcinoma subtypes show different patterns of metastatic behavior. Virchows Arch. 2017 Mar;470(3): 275-83.

8 Landry AP, Zador Z, Haq R, Cusimano MD. Reclassification of breast cancer: towards improved diagnosis and outcome. PLoS One. 2019 May; 14(5):e0217036.

9 Pusztai L, Mazouni C, Anderson K, Wu Y, Symmans WF. Molecular classification of breast cancer: limitations and potential. Oncologist. 2006 Sep;11(8):868-77.

10 Xiao W, Zheng S, Yang A, Zhang X, Zou Y, Tang H, et al. Breast cancer subtypes and the risk of distant metastasis at initial diagnosis: a population-based study. Cancer Manag Res. 2018 Nov; 10:5329-38.

11 Simmons C, Miller N, Geddie W, Gianfelice D, Oldfield M, Dranitsaris G, et al. Does confirmatory tumor biopsy alter the management of breast cancer patients with distant metastases? Ann Oncol. 2009 Sep;20(9):1499-504.

12 McAnena PF, McGuire A, Ramli A, Curran C, Malone C, McLaughlin R, et al. Breast cancer subtype discordance: impact on post-recurrence survival and potential treatment options. BMC Cancer. 2018 Feb;18(1):203.
13 de Dueñas EM, Hernández AL, Zotano AG, Carrión RM, López-Muñiz JI, Novoa SA, et al. Prospective evaluation of the conversion rate in the receptor status between primary breast cancer and metastasis: results from the GEICAM 2009-03 ConvertHER study. Breast Cancer Res Treat. 2014 Feb;143(3):507-15.

14 Allred DC. Commentary: hormone receptor testing in breast cancer: a distress signal from Canada. Oncologist. 2008 Nov;13(11):11346.

15 Kurbel S. Selective reduction of estrogen receptor (ER) positive breast cancer occurrence by estrogen receptor modulators supports etiological distinction between ER positive and ER negative breast cancers. Med Hypotheses. 2005;64(6):1182-7.

16 Navin N, Kendall J, Troge J, Andrews P, Rodgers L, McIndoo J, et al. Tumour evolution inferred by single-cell sequencing. Nature. 2011 Apr;472(7341):90-4.

17 Zustin J, Boddin K, Tsourlakis MC, Burandt E, Mirlacher M, Jaenicke F, et al. HER-2/neu analysis in breast cancer bone metastases. J Clin Pathol. 2009 Jun;62(6):542-6. 
18 Aurilio G, Monfardini L, Rizzo S, Sciandivasci A, Preda L, Bagnardi V, et al. Discordant hormone receptor and human epidermal growth factor receptor 2 status in bone metastases compared to primary breast cancer. Acta Oncol. 2013 Nov;52(8):1649-56.

19 Allred DC. The utility of conventional and molecular pathology in managing breast cancer. Breast Cancer Res. 2008;10(S4 Suppl 4):S4.
20 Amir E, Miller N, Geddie W, Freedman O, Kassam F, Simmons C, et al. Prospective study evaluating the impact of tissue confirmation of metastatic disease in patients with breast cancer. J Clin Oncol. 2012 Feb;30(6): 587-92.

21 Narod SA, Sopik V. Is invasion a necessary step for metastases in breast cancer? Breast Cancer Res Treat. 2018 May;169(1):9-23.

22 Chan CW, Law BM, So WK, Chow KM, Waye MM. Novel Strategies on Personalized Medicine for Breast Cancer Treatment: an Update. Int J Mol Sci. 2017 Nov;18(11):E2423.
23 Chen XS, Shen KW. [Multi-gene assay profiling testing and individualized therapy in breast cancer]. Zhonghua Wai Ke Za Zhi. 2017 Feb;55(2):99-103.

24 Olopade OI, Grushko TA, Nanda R, Huo D. Advances in breast cancer: pathways to personalized medicine. Clin Cancer Res. 2008 Dec;14(24):7988-99. 